Rev. Chil. Pediatr. 66 (5); 230-233, 1997

\title{
Síndrome de Goodpasture
}

\author{
Cristián Clavería R. '; Alejandro Donoso F.'; Cristián Valverde G. '; \\ Patricio Le Cerf $S^{2}{ }^{2}$; Camilo Boza W. ${ }^{3}$
}

\begin{abstract}
Resumen
El sincrome de Goodpasture es más trecuente en varones, adultos ćvenes. Se presenta una paciente de sexo Iemeriro de doce cños de edá con historia de palidez astenia y adinamic, expectoración hemoptoica y díficulrod respirakro rápidamente progresiva, anemia mitrocitica y falla renal aguca que evolucionó con insuficiencia respiratorio grave e irrevers ble secundaria a remorragia pulmonar masiva. Los exámenes anatomopatológ:cos mostraran hemosideros s pulmonor y gomerulonefritis crecéntico, comporibles con sindrome ce Goodposture.
\end{abstract}

[Palabras clave: hemosiderosis pulmonar, glomerulonefritis, sindrome de Goodpaslure.i

\section{Goodpasture's syndrome in a girl}

A twelve year ald lemale polieni presented with syslemic complainis o' malaise, ancrexia, respiratory distress.

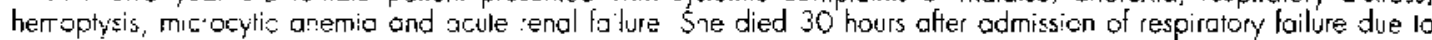
massive lung henorrcge. Postmortem examination showed pul nonory hemosiderosis and erecenric glomerulonephritis. Clinical and laboralory features support the diagnosis of Goadposture's syndrome which is lese frequently seen in young girls thon in young male adults.

(Key words: pulmonary' 'remosiderosis, glomeruionephrilis.?

El sîndrome de Goodpasture es una enfermedad autoinmune con clara preferencia por adultos jóvenes de sexo masculino (varones: mujeres $=6: 1$ ). Se expresa con hemoptisis secundaria a hemorragia alveolar y glomerulonefritis rápidamente progresiva, relacionadas con la presencia de depósitos de anticuerpos antimembrana basal alveolar y glomerular (reacción imnunológica tipo II). Las manifestaciones pulmonares son las predominantes y determinan la evolución de la enfermedad, pues corresponden con la principal causa de la muerte en estos pacientes. Existen pocas comunica-

I. Lnidad de Cuidados Intensivos Pediátricos. Hospital Dr. Sótero del Río.

2. Servicio de Anatomia Patológica. Hospital Dr. Sótero del Rio.

3. Interno de Medicina. Pontificia Universidad Católica de Chile. ciones sobre la afección en la literatura pediátrica internacional. Se describe la evolución de una paciente de doce años de edad que falleció a causa de ella, con el propósito de destacar sus manifestaciones clínicas y de laboratorio y enfatizar que esta enfermedad debe incluirse en el diagnostico diferencial de las hemorragias pulmonares difusas en niños y niñas.

\section{Caso Clínico}

Nin̄a de 12 affos. previamente sanc. Desde un mes antes de ingresar tenía tos, expectoración hemoptoica, disnea y palidez progresiva. En el hemograma, once días antes de ingresar, el hematocrito era $21 \%$, microcitosis moderada, 14500 leucocitos $\cdot \mathrm{mm}^{3}, 83 \%$ neutrófilos, concentracion normal de plaquetas, velocidad horaria de sedimentación globular (VHS) $1.54 \mathrm{~mm} / \mathrm{h}$. Se irato ambulatoriamente con antibióticos y hierro por vía oral. Cuarenta y ocho horas antes del ingreso se agreg 6 fiebre (tuvo hasta $39.5^{\circ} \mathrm{C}$ rectal), astenia y adinamia. disnea en reposa, ortopnea y 
aumento de la expectoración. Jngritsó - a la unidad de euidados intelasivos pediatreces-cousciene. con gran palidez,

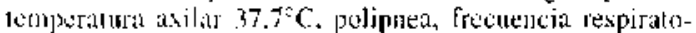

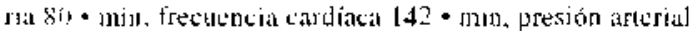
I thit mnנHg En los pulmones se registaron ahundantes cripitos, ausencia de murmullo vesiculial en los dos tercios inferioles de] hemitorax derecho. Latidos cardíacos hiperdinámions, ritno reguar, sin soples. No sc encontraron sișnos de visceromegalia es el abdomen. Hemalocrto $96, \mathrm{PaO} / \mathrm{FiO}, \mathrm{LBO}, \mathrm{pH} 7.17$ y exceso de base -19.5 en la sangre arterial; nitrógeto urẹico sanguineo $\$ 51 \mathrm{~kg} / \mathrm{dl}, \mathrm{K}$ plasmáticu 6,$]$ mEq/t (liblá). En la radiografias ale tórax se vecan infiltsados alveolares difusos extensos, mús acentuados en el pulmón derecho. Se conectó a un ventilador mecúnico en la modalidad controlada por presión, empleando. descle t cotnjen 20 , presión media de vía aćres (PMVA) de

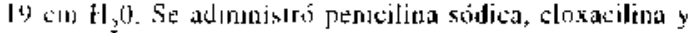
celotaximiz y metilprednisolona intravenosa el "pulsos". soluciones de eristaloides. 1ranslisión de glóbulos rojos. nodicamentos shoroficos. sin eonseguir mojuría de la fintion renal en las primerat seis hojas, observaludose maís

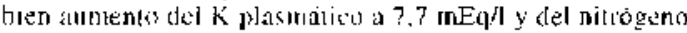
ureico a 12+ nagld. por lo que se inicio hemotiluacicin

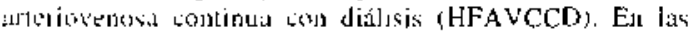
horas siguimls's la funcion pulmonar se detcrionó progresi-

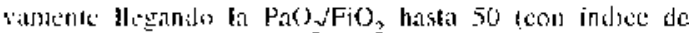

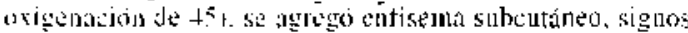
de ncimoloras hidaleral y harturauma generalizado en las radiografías de tórax. Falleció 30 h despućs de ingresar. sin haber sido posible realizar bjopsia renal.

En la necropsia se encontjaron hemoragias pulmonares difusas extensas, recientes: henorragias intrablveolates extensas que comprometian los lumenes bronquiolares $y$ bromquiales, iton numerosos macrófagos que contenian hemosidcrina citoplasınatica (siderrilagos) y hemosiderosis pulmonar (Figura l). Glomerulonefritis crecéntica difusa $y$ seneralizada. con proliferaction extracapilar en Ja gran mayoría de los glomérulos, muchos de los cuales se encuentrain olssoletos (aproximadatzente 20\%) (fígura 2), La inmenohistoquínica (mélodo ABC) empleando anticuerpos monoclonales antilga mostró posilividird lineal ti el nesangio glomerular $y$ en las paredes alveolares. Los hallazgos descritos son compatibies con sindrome de Goodpasture

\section{Comentario}

El trasiorno que presentó esta paciente coresponde a los denominados síndrome hemorrásico pulmonar y su subclasificación como síndrome pulmonar-renal. Los hechos clínicos, de laboratorio y la necropsia permiten configurar el síndrome de Goodpasture, destacando su

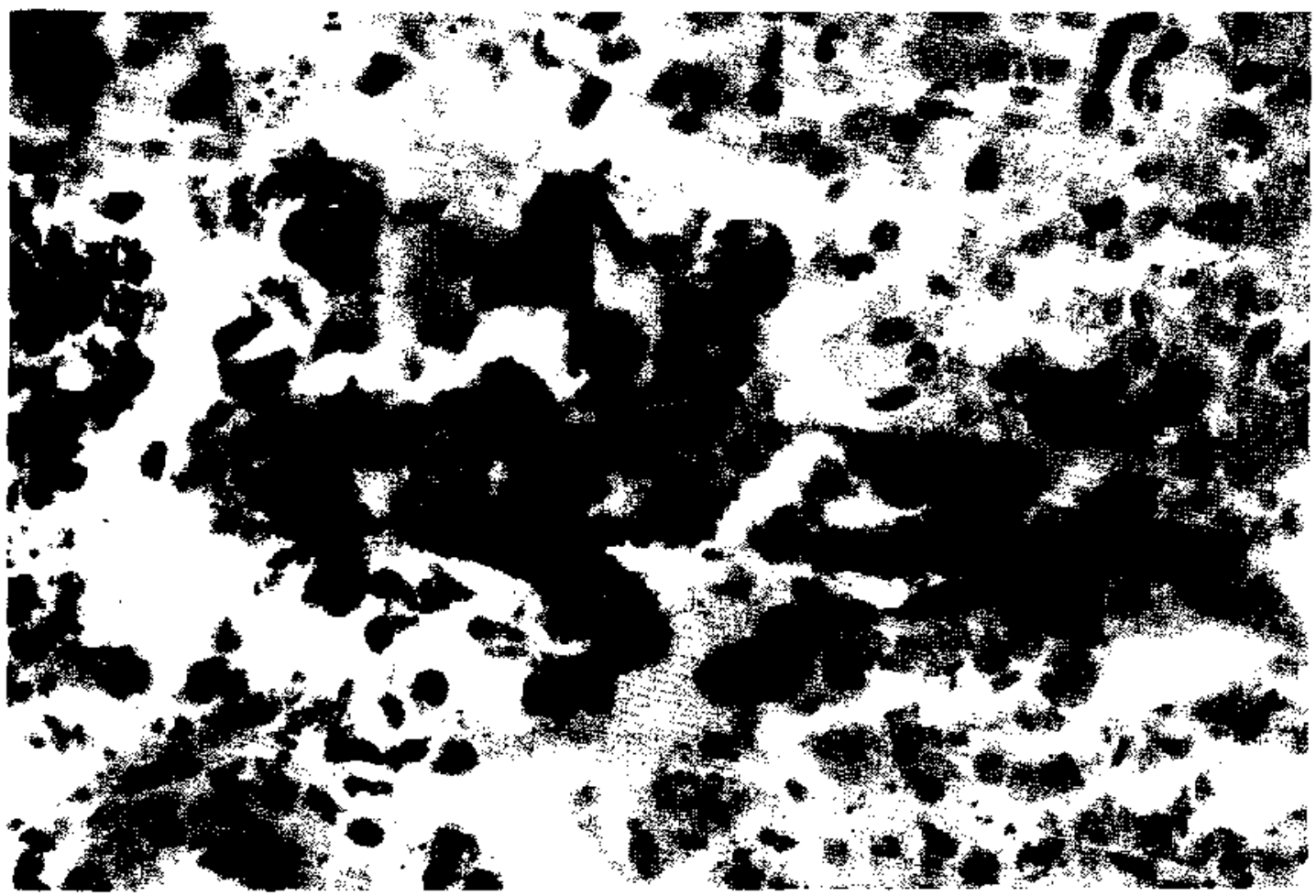

kigura 1: Aacrilagos eon henwosiderina en el lumen alveolar (daul de Prusia, 400x). 


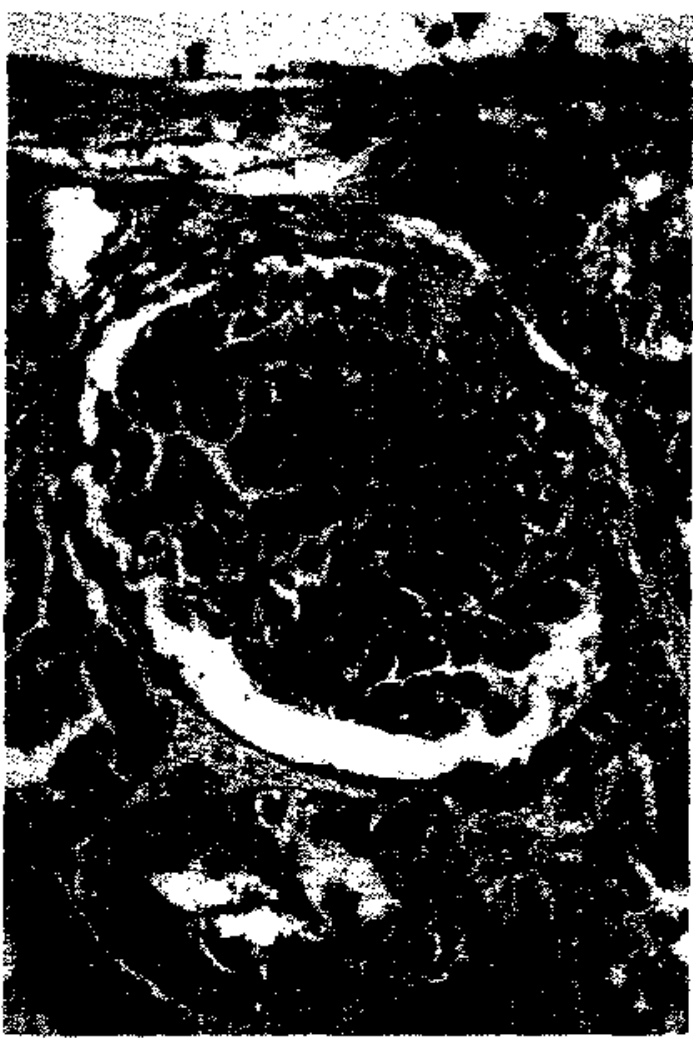

Figura 2: Gilırectulisnclijtis encénlico ilifusa con proljle-

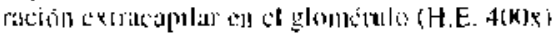

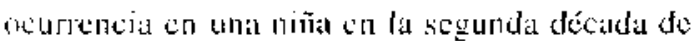
la vida.

I.a descripcion orizinal de la asociación de hamoragia pulnomar extensa y compromiso renal tue hechat por Goudpasture on 19191. En 1958 Stanton y Tange ${ }^{2}$ utidiziron el cérmino "sindrome de Goodpasturc" all reportar la asocliaciśn entre hemorragia pulmonar y glomerulonefritis, el cual continúa siendo aplicado a pacientes con hemoptisis, signos radiológicos de culermedad infilltaliva pulmonar difusa, anemia y falla renal eon daño glomerular. En 1964 se describicron depósilos lineales de inmunoglobulinas en al riñón de pacientes con una forma similar de presentacion ${ }^{3}$. Pustcriotmente se delerminó la presencia de depósilos de inmunuglobulinas сл los puimones de pacientes con eske sindrome ${ }^{+}$y que cllos correspondiat a anticuerpos conira la membrana basal glome- rular (MBG), ubicables circulando en el suero y fijos en los rejiclos 5 .

E:I posible antígeno es el colágeno tipo IV de la membrata basal glomerular, que gatillaria una respucsta inmunitaria que incluiría síntesis de anticuerpos contra la membrana basal ${ }^{6}$. El desarrotlo de capacidad antigénica scría secundario a infecciones virales como la producida por virus influenza o cualquice otro tipo de injuria que dañe la membrana basal glomerujar, como inhalación de solventes volátiles de tipo hidrocarburos e ingestión de penicilamina ${ }^{7.8}$. La lcoría cxelusivamente inmunológica sobre la patogenia del sindrome de Goudpasture lienc debilidades. pues en algunos pacientes que corresponden al síndrome, no ha sido posible demostrar anticuerpos contra la membrana basal en el suero y en los depósitos de material inmunitario en riñones y pulmones ${ }^{9} .10$.

La hemorragia alveolar masiva lue la causa de muerte en $30 \%$ de los pacientes de una serie ell que la cdad variaba de 16 a 61 años, y la subrevida. promedion. desde el diagnóstico, fue 15 semanas". Usuafmentc ba hemoptisis precede a la falla renal, pro esta suele ser rápidamente progresiva. En nuestra paciente, consjderando la rapida cvolución, no lue posible determinar anticuespos contra la membrana basal del gromérulo, cuya medición es útil en el diagnóstico y paral orientar el tratamiento con inmumosupresores, drogas cilolóxicas y plasmatéresist?

La plasmaféresis se emplea con el propósito de reducir la cantidad de anticuerpos circulantes contra la membrana basal y ha producido efeclos beneficinsos sobre el curso clínicio de la enformedad. particularmente si se aplica precozmente. to que no correspondia en este caso. Además para usar este procedimiento el paciente debe estar hemodinamicamente estable ${ }^{13}$.

E) diagnóstico dilerencial del síndrome de Goodpasture incluye las dilerentes formas de vasculitis sistérnica que pucden comprometcr pulmón y riñón, como la granulomatosis de Wegener, el sindrome de Churg-Sirauss, la poliartcritis nodosa, ha esclerosis sistémica progresiva y el lupus critematoso sistémico. Todas ellas son también infrecuentes en niños, pero se pueden asociar con hemoplisis, ta que taras veces es significativa. La alteración de la función renal es da evolución más lenta que cn la paciente que comentamos, La biopsia renal sigue 
siendo la principal herramienta para hacer un diagnóstico cortecto en la evaluación de los sindromes pulmón-riñón ${ }^{14}$.

\section{Referencias}

1. Guskipusture $E$ W: The significance of certain pulmonary lesions in relation to the etiology of influenza. Ani J Med Sii ]919: 158: $863-870$.

2. Situm HC. Tangs , $D$ : Goodpasture's syndrome (putmonity hemorthige associated with glomerulo. nephritis). Aust Ann Med 1958: 7: 132-144.

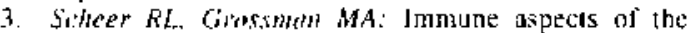
glomerulonephritis associared with pulnonary hemorrhags:. Ann Intern Med 1964: 60: 1009-102!

4. Srurgill $B C$. Westervell $F B$ : Immunoflubescence studjes in a case of Goodpasturc's syndronis. JAMA 1965: 194: 914.916.

5. Thomas HAM WI. Inin RS: Classification of d]fuse intrapulmonary hemorrhage. Chest 1975; 68: 483-484.

6. Wite $G$. Thimpl $R$ : Study on the nature of the Goodpasture antigen using a basement nembraneproducing mouse rumor. Clin Exp Unmunol 1980: 39: $733-738$

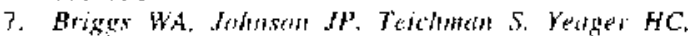
Wifson CB: Antiglomerulat basemenet monbrane anribody-mediared glomerulonephritis and Goodpasture's syndrome. Modicine (Baltimore) 1979; $58: 348$. 361 .

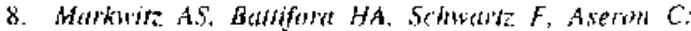
Immunological aspects of Goodpasture's syndrome. Clin Exp Iıntnunol 1968: 3: 585-591.

9. Buyce NW, Hotdsworth SR: ldiopathic Goodpasture's syndtome: fatal pulmonary hemorthage and crescentic glomerulonephritis in the absente of immune-resctant Jeposition. Nephron 1986: 44: 22-25.

10. Cohen AH. Bowder WA, Sinukel E, Giassaik RJ: Crescentic glomerulonephritis: immune vs nonimmune mechanisms. Am I Nephrol 1981: J: 78-83.

II. Bewoit FL. Rulon DB, Theil GB, Donlan PD, Watten $R H$ : Goodpasture's syndrome: clinico puthologic entity. Am J Med 1964; 37: 424-444.

12. Conk 10: Therapeutic plostnapheresis (plasnia ex(hange). Edited by Levin DL. Morriss FC. Essentials of Pediatric Inrensive Care, Quality Medical Publishing. Inc. St. Louis Missouri, 1990: 978-983.

13. Johnson IP, Whitwart W, Briggs WWA, Wilson CB: Plasmapheresis and immunosuppressive agentes in anibasement membrane antibody-induced GoodpasLure"s syndrorae. Am I Med 1978; 64; 354-359.

14. lefebure $C$. Lamber $M$, Pirson Y: Pulmonary-renal syndrome: diagnostic and therapcutic statcgy. Actn Clin Belg 199.5; 50: 94-102

\section{AVISO A LOS AUTORES}

Por acuerdo del Comité Editorial, la Revista Chilena de Pediatría devolverá sin tramitar todos los trabajos que no den estricto cumplimiento al Reglamento de Publicaciones y a las Instrucciones de los Autores que se editan en cada número de la Revista. 